\title{
Parenchymal Congestion Is Important for Rapid Regeneration of the Future Liver Remnant Following the ALPPS Procedure
}

\author{
DAISUKE KAWAGUCHI ${ }^{1}$, YUKIHIKO HIROSHIMA ${ }^{1}$, KENICHI MATSUO ${ }^{1}$, \\ ITARU ENDO $^{2}$, KEIJI KODA ${ }^{1}$ and KUNIYA TANAKA ${ }^{1}$ \\ ${ }^{1}$ Department of Surgery, Teikyo University Chiba Medical Center, Chiba, Japan; \\ ${ }^{2}$ Department of Gastroenterological Surgery, \\ Yokohama City University Graduate School of Medicine, Yokohama, Japan
}

\begin{abstract}
Aim: To evaluate whether the congested area that develops in associating liver partition with portal vein ligation for staged hepatectomy (ALPPS) contributes to rapid future liver remnant (FLR) hypertrophy. Patients and Methods: Eight patients undergoing liver partition and right portal vein $(R P V)$ ligation within the FLR in the first operation of ALPPS were compared with eight patients undergoing RPV embolization in the FLR as the first operation of classical two-stage (CTS) hepatectomy. Results: Extrapolated kinetic growth of the FLR in ALPPS was 32.7 $\pm 18.7 \mathrm{ml} /$ day, 7.8-times that in CTS $(4.2 \pm 2.0 \mathrm{ml} /$ day, $p=0.001)$. Extrapolated kinetic reduction in volume of the ventral aspect of the right paramedian sector, which became congested after the first procedure in ALPPS, was 19.8 $\pm 11.6 \mathrm{ml} /$ day, 11-times that in CTS $(1.8 \pm 1.3 \mathrm{ml} /$ day, $p=0.001)$. Conclusion: Production of a congested area within the deportalized liver may contribute importantly to rapid FLR hypertrophy during ALPPS.
\end{abstract}

Liver resection remains the only potentially curative option for patients with colorectal liver metastases. Surgeons continue to expand the limits of "resectable" disease by performing extensive liver resections that challenge both the oncologic and technical definitions of resectability. Within this context of extended resection, postoperative liver failure arising from insufficient function of the future liver remnant (FLR) remains a major concern, especially in patients who have undergone extensive preoperative chemotherapy. Multiple strategies to increase the size of the FLR and, thereby, reduce the risk of postoperative liver failure have been applied.

Correspondence to: Kuniya Tanaka, MD, Department of Surgery, Teikyo University, Chiba Medical Center, 3426-3 Anesaki, Ichihara, Chiba, 299-0111, Japan. Tel: +81 436621211, Fax: +81 436613961, e-mail: U17-92TS@med.teikyo-u.ac.jp

Key Words: ALPPS, congestion, liver regeneration.
A new variant of portal vein (PV) occlusion associated with staged hepatectomy was recently found to enhance volume increase in the FLR (1). This approach combines liver partition with PV ligation (PVL), followed by a second operation to remove the deportalized, diseased part of the liver. The acronym "ALPPS" (Associating Liver Partition with Portal Vein Ligation for Staged Hepatectomy) has been proposed to describe this complex procedure (2). Initial experience suggested that the volume increase after ALPPS is more rapid than that obtained with previous techniques, such as PV embolization (PVE) followed by hepatectomy or classical two-stage (CTS) hepatectomy, allowing for removal of the diseased part of the liver within only 1 to 2 weeks after liver partition $(1,3)$. This rapid regenerative response would seem to offer significant advantages, given that major tumor progression is unlikely during this short period and that rapid regeneration could permit a second hepatectomy before development of troublesome adhesions. Additionally, the shortened surgical treatment period may allow faster patient recovery, possibly permitting earlier resumption of chemotherapy (2).

The exact mechanisms underlying the remarkable regenerative response of the FLR in ALPPS are still unclear. After the first resection in ALPPS, extent of the area with inflammatory injury is thought to contribute to intense stimulation of liver regeneration. ALPPS also produces a completely devascularized area, such as segment (S) 4 in the right trisectorectomy, resulting in early shrinkage of that area with compensatory FLR hypertrophy (4); however, such a devascularized area can become a necrotic focus of sepsis. Another possible stimulus to regeneration involves the area of congestion induced by interruption of hepatic vein branches during parenchymal partition. This area of venous congestion may develop considerable atrophy, resulting in compensatory hypertrophy of the FLR. When the middle hepatic veins (MHV) are sacrificed during liver partition within S4, a part of S4 and the ventral aspect of the right 
paramedian sector (RPS) $(5,6)$ become congested following right (rt.) hepatectomy extended to a part of S4. We believe that this congested area is critical for FLR hypertrophy in ALPPS. However, little information is available regarding how closely the congested area is related to the increase in FLR volume.

We, therefore, set out to clarify whether the congested area produced by the ALPPS treatment contributes importantly to rapid FLR hypertrophy, especially in terms of serial changes in both morphologic and functional liver volumes.

\section{Patients and Methods}

Patients. Eight consecutive patients treated with ALPPS between April 2013 and September 2014 were compared with eight others who underwent CTS hepatectomy with PVE between August 2012 and April 2013. For all patients in the ALPPS group, the MHV were ligated and divided during transection within S4 in rt. hepatectomy.

Imaging. Sequential changes in morphologic and functional volume of the FLR and other liver areas were assessed by multi-detector computed tomography (CT) and technetium-99m galactosyl human serum albumin (99mTc-GSA) single-photon emission computed tomography (SPECT) scintigraphy. Results were compared between the 2 groups.

Surgical procedure. In the CTS hepatectomy, most first procedures involved resection of metastases from the left hemiliver anticipated to become the FLR (tumor-clearing resection) (7), immediately followed by performance of PVE for the right hemiliver (Figure 1a). A catheter was inserted into a portal vein through an ileocolic vein and the portal venous branches of the hemiliver targeted for resection were embolized. The embolic material was a mixture of gelatin pellets and oleic acid monoethanol amine. Later, at the second operation, extended right hepatectomy (extended to a part of S4) was performed. While timing of the second resection depended on degree of liver hypertrophy or duration of chemotherapy, we generally measured volume by CT 3 weeks after the first operation and performed the second hepatectomy 4 weeks after the initial hepatectomy with PVE. This second resection was performed only when it could be curative.

In the ALPPS procedure, once the liver was mobilized, total liver partition to the level of the inferior vena cava (IVC) was carried out. During right (rt.) hepatectomy, liver partition was performed between main and right portal fissures (within S4), accompanied by MHV division. Tumor-clearing surgery was performed in the FLR after which the portal vein of the diseased right hemiliver was ligated (Figure 1b). Seven days after the first operation, CT was performed to determine FLR volume. If this volume was considered sufficient and the patient was stable, extended right hepatectomy (extended to a part of S4) was performed.

Final status of the FLR is highly similar in these 2 procedures. However, the ventral RPS becomes both deportalized and congested in ALPPS but becomes deportalized only without congestion after the first operation in the CTS treatment. Couinaud's terminology was used to designate operative procedures and liver anatomy (8).
Liver volume and functional liver volume evaluation. CT images were obtained preoperatively and at 7 days after the first surgical procedure, using multidetector CT ((MDCT); Toshiba Medical, Tokyo, Japan) to determine volumes of the FLR and other liver areas in the ALPPS group. In the CTS hepatectomy group, MDCT was performed preoperatively and 3 weeks after the first procedure. Functional liver volume was calculated by $99 \mathrm{mTc}-\mathrm{GSA}$ SPECT/CT using a Symbia T16 scanner (Siemens, Munich, Germany). Reconstructed SPECT and CT images were converted into the DICOM format and then transferred to SYNAPSE VINCENT (Fujifilm, Tokyo, Japan) for fusion imaging. After fusion images were reconstructed in 2 views (axial and coronal slices), 3dimensional (3D) fused images were reconstructed, providing volume-rendering SPECT and CT images. The \% functional volume was estimated by scintillation counting for the FLR and other areas relative to the total count for the entire liver. In this study, the ventral RPS was defined in a transverse CT image as the area between the MHV and the alleged anterior portal fissure (6), as determined by a line connecting the main trunk of the right anterior portal pedicle with the IVC (Figure 1c, d).

Perioperative factors analyzed. Postoperative complications were evaluated according to the Dindo-Clavien classification (9). Posthepatectomy liver failure was defined as postoperative deterioration in the ability of the liver to maintain its synthetic, excretory and detoxifying functions, as characterized by increased prothrombin time (PT) and concomitant hyperbilirubinemia on or after postoperative day 5. Post-hepatectomy liver failure was classified as grade A, B or C according to the International Study Group of Liver Surgery definitions (10).

Statistical analysis. Continuous data are expressed as mean $( \pm \mathrm{SD})$ or median (range) and were analyzed using the Mann-Whitney $U$ test. The $\chi^{2}$ test or Fisher's exact test was used for analysis of categorical variables. A difference was considered significant when the two-sided $p$-value was below 0.05 .

Ethical considerations. The study protocol was approved by the Institutional Ethics Committee at Teikyo University, Japan (notice of approval of IRB protocol number, Teirin 14-119). Written informed consent was obtained from all patients involved.

\section{Results}

Patients' characteristics. No significant differences in patients' background were observed between groups. The 16 patients included in this study consisted of 9 men (56\%) and 7 women (44\%). Their diagnoses were liver metastases from colorectal cancer or from pancreatic neuroendocrine tumor. Their median age was 68 years (range $=62-78$ ). Liver metastases showed a bilobar distribution in all patients. The median number of metastases at diagnosis was 11 (range $=4$ 19). The median maximum size of metastases was $35 \mathrm{~mm}$ (range $=8-168$ ). The final hepatectomy procedure was extended rt. hepatectomy (extended to S4) with tumorclearing resection from the FLR in all patients both with ALPPS and with CTS hepatectomy. All patients in both groups received preoperative chemotherapy (Table I). 
a

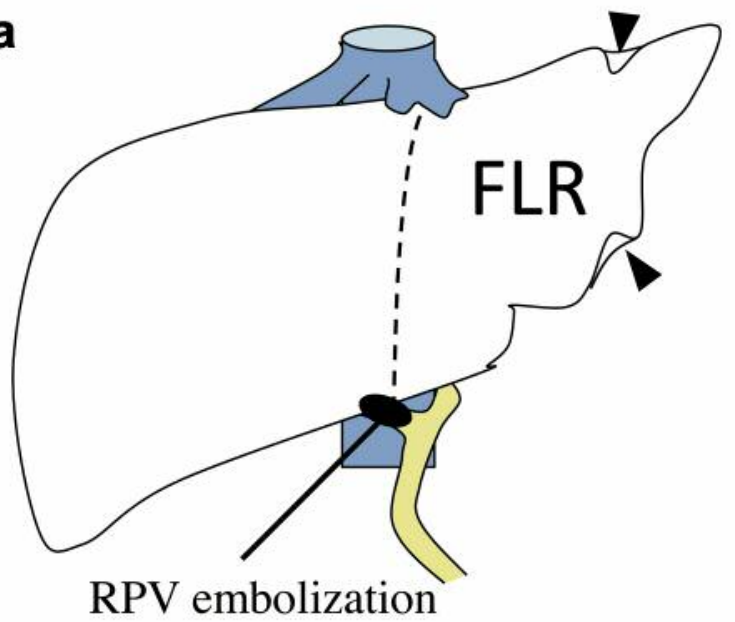

C

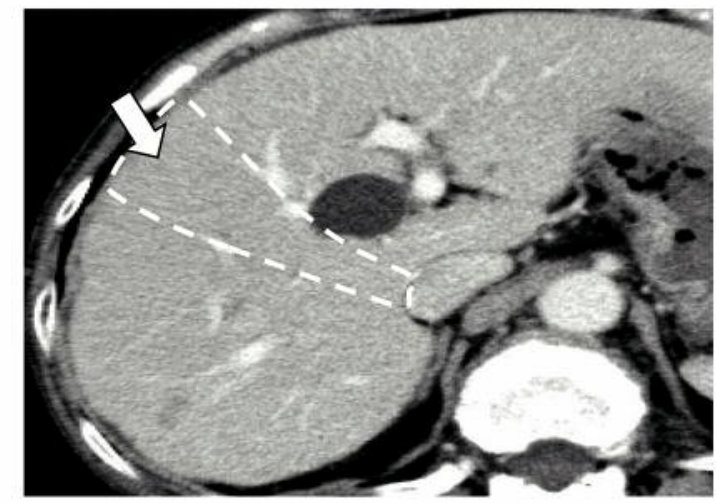

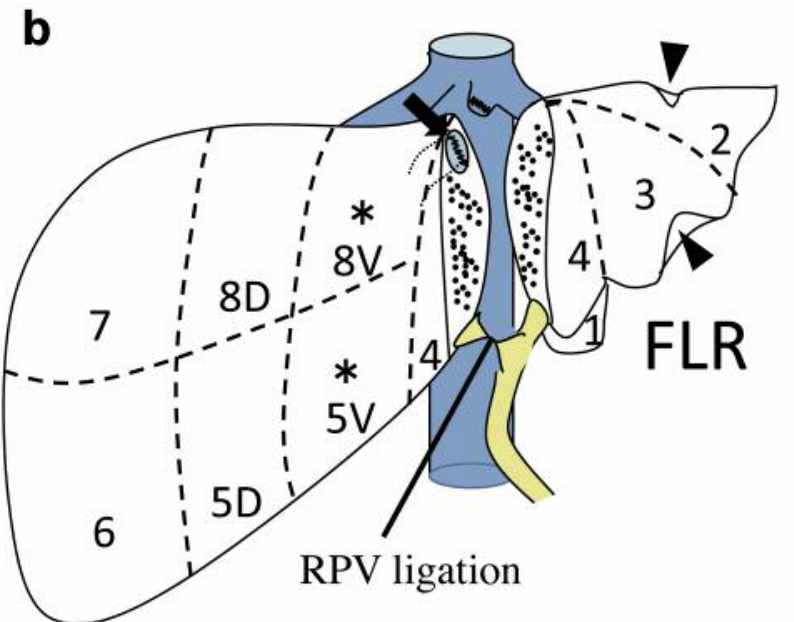

d

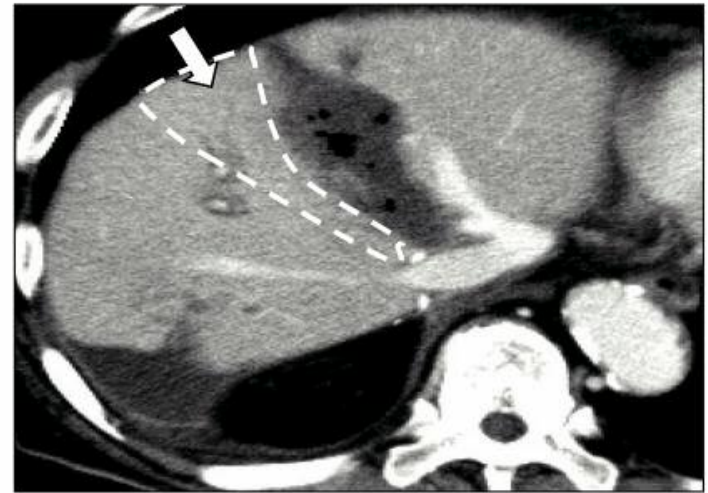

Figure 1. Diagrammatic description and computed tomography (CT) depiction of classical 2-stage (CTS) hepatectomy and associating liver partition with portal vein ligation for staged hepatectomy (ALPPS). After the first stage of the CTS hepatectomy (a), resection of metastases from the left hemiliver anticipated to become the future liver remnant (FLR) was performed (arrowhead), followed by performance of portal vein (PV) embolization for the right (rt.) hemiliver. After the first stage of ALPPS (b), complete tumor resection in the FLR was performed (arrowhead). The middle hepatic veins (MHV) were ligated at the trunk (arrow) and divided during transection of segment (S) 4 in extended rt. hepatectomy (extended to S4). The rt. PV was sutured. The ventral aspect of the rt. paramedian sector (RPS) became congested (asterisk). CT findings after first hepatectomy, at 3 postoperative weeks in CTS hepatectomy (c) and on postoperative day 7 in ALPPS (d). Ventral RPS (arrow) and partition line are shown. The ventral RPS showed congestion and atrophy in ALPPS. RPV, right portal vein.

As for operative factors, no significant differences were observed between groups. Morbidity and mortality rates were comparable between groups; 1 patient in the CTS group died. According to the Clavien-Dindo classification, no class IIIB or greater severe complication was observed in the ALPPS group. In the CTS group, a class IIIB or more severe complication was observed in 1 patient each at the first or second procedure. Liver failure (grade B) occurred in 1 patient $(12.5 \%)$ after the second procedure of the CTS hepatectomy (Table II).

Change of volume, kinetic growth and functional volume. The deportalized rt. liver was further divided into ventral RPS and other areas (dorsal RPS plus rt. lateral sector (RLS)). Changes in volume of the FLR and the other areas during treatment were calculated and compared between the 2 groups.

Before the first procedure, FLR volume was $356.4 \pm 54.4 \mathrm{ml}$ (median=315.6; range $=300.3-475.7$ ) in the ALPPS group and $322.1 \pm 48.7 \mathrm{ml}(323.0 ; 261.1-397.0)$ in the CTS group $(p=0.204)$. After the first procedure, FLR volume was $569.6 \pm 156.1 \mathrm{ml}(558.1 ; 365.8-815.7)$ in the ALPPS group and $427.4 \pm 91.7 \mathrm{ml}(416.6 ; 290.5-541.7)$ in the CTS group $(p=0.043)$. Extrapolated kinetic growth for the ALPPS group was $32.7 \pm 18.7 \mathrm{ml} /$ day, which was 7.8 times that in the CTS group $(4.2 \pm 2.0 \mathrm{ml} / \mathrm{day}, p=0.001)$. As for functional volume, $\%$ functional FLR volume relative to total liver volume before the 
Table I. Patients' and tumor characteristics.

\begin{tabular}{|c|c|c|c|}
\hline Variables & $\begin{array}{c}\text { ALPPS } \\
(\mathrm{n}=8)\end{array}$ & $\begin{array}{l}\text { CTS } \\
(\mathrm{n}=8)\end{array}$ & $p$-Value \\
\hline \multicolumn{4}{|l|}{ Patient-related } \\
\hline Age, years & $68(62-78)$ & $66(65-74)$ & 0.887 \\
\hline \multicolumn{4}{|l|}{ Gender } \\
\hline Male & $4(50 \%)$ & $5(62.5 \%)$ & \multirow[t]{2}{*}{0.614} \\
\hline Female & $4(50 \%)$ & $3(37.5 \%)$ & \\
\hline \multicolumn{4}{|l|}{ Primary disease } \\
\hline $\mathrm{CRC}$ & $7(87.5 \%)$ & $8(100 \%)$ & \multirow[t]{2}{*}{0.302} \\
\hline NET & $1(12.5 \%)$ & 0 & \\
\hline \multicolumn{4}{|l|}{ Liver-related } \\
\hline Timing & & & \multirow{3}{*}{0.522} \\
\hline Synchronous & $7(87.5 \%)$ & $6(75 \%)$ & \\
\hline Metachronous & $1(12.5 \%)$ & $2(25 \%)$ & \\
\hline \multicolumn{4}{|l|}{ Distribution } \\
\hline Bilobar & $8(100 \%)$ & $8(100 \%)$ & $>0.999$ \\
\hline Number & $11(4-19)$ & $11(5-18)$ & 0.865 \\
\hline Maximum tumor size, $\mathrm{mm}$ & $22(11-34)$ & $31(8-168)$ & 0.225 \\
\hline \multicolumn{4}{|l|}{ Extrahepatic disease } \\
\hline Present & $1(12.5 \%)$ & $1(12.5 \%)$ & $>0.999$ \\
\hline \multicolumn{4}{|l|}{ Treatment-related } \\
\hline \multicolumn{4}{|l|}{ Prehepatectomy CTx } \\
\hline Performed & $8(100 \%)$ & $8(100 \%)$ & $>0.999$ \\
\hline Number of cycles & $18(6-44)$ & $6(6-42)$ & 0.333 \\
\hline Number of lines & $2(1-6)$ & $1(1-6)$ & 0.258 \\
\hline \multicolumn{4}{|l|}{ PVE/PVL } \\
\hline Performed & $8(100 \%)$ & $8(100 \%)$ & $>0.999$ \\
\hline \multicolumn{4}{|l|}{ Curability } \\
\hline $\mathrm{R} 0 / 1$ & $8(100 \%)$ & $8(100 \%)$ & $>0.999$ \\
\hline $\mathrm{R} 2$ & 0 & 0 & \\
\hline
\end{tabular}

Values of continuous variables are medians. Ranges are shown in parentheses. ALPPS, Associating liver partition with portal vein ligation for staged hepatectomy; CTS, classical 2-stage hepatectomy; CRC, colorectal cancer; NET, neuroendocrine tumor; PVE, portal vein embolization; PVL, portal vein ligation; CTx, chemotherapy.

first procedures was $40.3 \pm 9.9 \%(37.5 ; 29.3-58.3)$ in the ALPPS group and $34.0 \pm 5.9 \%(36.1 ; 23.5-39.5)$ in the CTS group $(p=0.143)$. After the first procedure, $\%$ functional FLR volume in the ALPPS group was $48.6 \pm 11.7 \%(53.3 ; 32.8-61.1)$ and $51.8 \pm 8.3 \%(50.7 ; 39.8-69.3)$ in the CTS group $(p=0.546)$.

Before the first procedure, ventral RPS volume in the ALPPS group was 221.2 $\pm 78.7 \mathrm{ml}$ (median=192.5; range=126.6-360.5) and $223.6 \pm 44.7 \mathrm{ml}(218.5 ; 169.3-288.7)$ in the CTS group $(p=0.951)$. After the first procedure, ventral RPS volume was $84.3 \pm 59.4 \mathrm{ml}(91.7 ; 16.8-182.8)$ in the ALPPS group and $181.3 \pm 52.9 \mathrm{ml}(170.2 ; 120.6-271.1)$ in the CTS group $(p=0.004$; Figure $2 \mathrm{a})$. The extrapolated kinetic decrease in volume of the ventral RPS for ALPPS was $19.8 \pm 11.6 \mathrm{ml} /$ day, which was 11 times greater than for the CTS group $(1.8 \pm 1.3$ $\mathrm{ml} /$ day, $p=0.001$; Figure 2b). As for functional volume, $\%$ functional ventral RPS volume before any procedure was $20.1 \pm 7.1 \%(22.3 ; 7.7-29.9)$ in the ALPPS group and 21.6 $\pm 5.8 \%$
Table II. Operative variables and short-term outcome.

\begin{tabular}{|c|c|c|c|}
\hline Variables & $\begin{array}{l}\text { ALPPS } \\
(\mathrm{n}=8)\end{array}$ & $\begin{array}{l}\text { CTS } \\
(\mathrm{n}=8)\end{array}$ & $p$-Value \\
\hline \multicolumn{4}{|l|}{ Operative variables } \\
\hline \multicolumn{4}{|c|}{ Duration of operation, min } \\
\hline First Hx & $415(273-510)$ & $342(255-454)$ & 0.878 \\
\hline Second $\mathrm{Hx}$ & $207(130-329)$ & $521(287-878)$ & 0.263 \\
\hline \multicolumn{4}{|l|}{ Blood loss, $\mathrm{mL}$} \\
\hline First Hx & $504(337-787)$ & $317(49-378)$ & 0.164 \\
\hline Second $\mathrm{Hx}$ & $353(100-680)$ & $666(303-960)$ & 0.203 \\
\hline \multicolumn{4}{|c|}{ Blood transfusion performed } \\
\hline First Hx & $1(12.5 \%)$ & 0 & 0.302 \\
\hline Second $\mathrm{Hx}$ & $1(12.5 \%)$ & $3(27.5 \%)$ & 0.248 \\
\hline \multicolumn{4}{|l|}{ Postoperative outcome } \\
\hline \multicolumn{4}{|l|}{ Morbidity (Any) } \\
\hline First $\mathrm{Hx}$ & 0 & $1(12.5 \%)$ & 0.302 \\
\hline Second $\mathrm{Hx}$ & $1(12.5 \%)$ & $3(37.5 \%)$ & 0.248 \\
\hline \multicolumn{4}{|l|}{ Morbidity } \\
\hline \multicolumn{4}{|c|}{ (Clavien-Dindo $\geq$ IIIB) } \\
\hline First Hx & 0 & $1(12.5 \%)$ & 0.302 \\
\hline Second $\mathrm{Hx}$ & 0 & $1(12.5 \%)$ & 0.302 \\
\hline \multicolumn{4}{|l|}{ Mortality ( $\leq 90$ days) } \\
\hline First Hx & 0 & 0 & $>0.999$ \\
\hline Second $\mathrm{Hx}$ & 0 & $1(12.5 \%)$ & 0.302 \\
\hline $\begin{array}{l}\text { Total length of } \\
\text { hospital stay, days }\end{array}$ & $26.7(19-57)$ & $34.1(14-96)$ & 0.126 \\
\hline
\end{tabular}

Values of continuous variables are medians. Ranges are shown in parentheses. ALPPS, Associating liver partition with portal vein ligation for staged hepatectomy; CTS, classical 2-stage hepatectomy; Hx, hepatectomy.

(21.1; $15.7-32.9)$ in the CTS group $(p=0.630)$. After the first procedure, $\%$ functional ventral RPS volume was $10.1 \pm 4.0 \%$ $(11.1 ; 2.1-14.3)$ in the ALPPS group and $18.6 \pm 4.9 \%$ (17.8; 11.125.8 ) in the CTS group ( $p=0.002$; Figure $2 c$ ).

Before the first procedure, the volume of dorsal RPS+RLS in the ALPPS group was $359.2 \pm 118.1 \mathrm{ml}(345.5$; 192.4528.4); in the CTS group, this was $394.1 \pm 87.7 \mathrm{ml}$ (383.2; $271.2-536.3 ; p=0.511)$. After the first procedure, dorsal RPS+RLS volume in the ALPPS group was $297.6 \pm 62.9 \mathrm{ml}$ (327.5; 160.6-500.2); in the CTS group, this was $327.5 \pm 114.2 \mathrm{ml}(289.5 ; 216.1-379.1 ; p=0.528$; Figure 3a). The extrapolated kinetic decrease in volume of the dorsal RPS+RLS for ALPPS was $4.5 \pm 3.1 \mathrm{ml} / \mathrm{day}$, which was not significantly different from that in the CTS group $(3.9 \pm 1.7$ $\mathrm{ml} /$ day, $p=0.636$; Figure $3 \mathrm{~b}$ ). As for functional volume, $\%$ functional dorsal RPS+RLS volume before any procedure was $39.6 \pm 8.8 \%(38.3 ; 29.6-56.1)$ in the ALPPS group and $44.3 \pm 7.5 \%(44.3 ; 34.5-59.4)$ in the CTS group $(p=0.263)$. After the first procedure, \% functional dorsal RPS+RLS volume in the ALPPS group was $39.3 \pm 11.3 \%$ (36.2; 26.5$57.8)$; in the CTS group, this was $31.0 \pm 12.7 \%$ (28.3; 19.7$59.7 ; p=0.186$; Figure $3 \mathrm{c}$ ). 
a. volume

(ml) 250

200

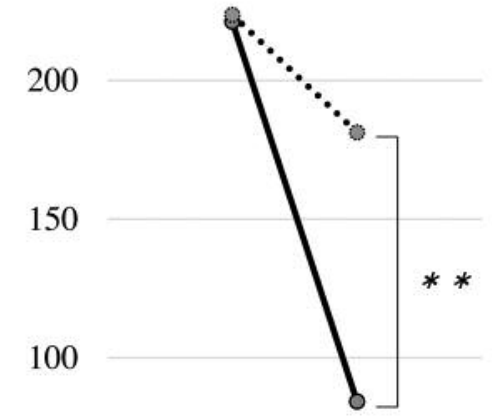

\begin{tabular}{ccc}
50 & pre & post \\
\cline { 2 - 3 } 2-staged & $223.6 \pm 44.7$ & $181.3 \pm 52.9$ \\
\hline ALPPS & $221.2 \pm 78.7$ & $84.3 \pm 59.4$
\end{tabular} c. Functional volume

(\%) 25

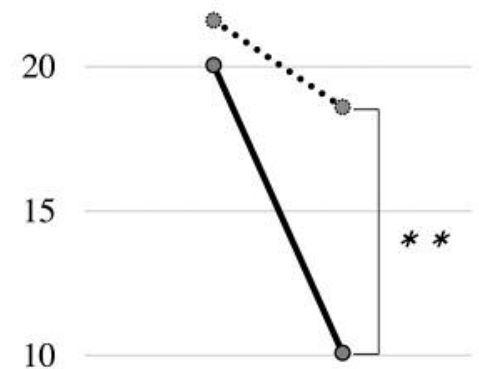

\begin{tabular}{ccc}
5 & pre & post \\
2-staged & $21.6 \pm 5.8$ & $18.6 \pm 4.9$ \\
\hline ALPPS & $20.1 \pm 7.1$ & $10.1 \pm 4.0$
\end{tabular}

\section{b. Kinetic changes}

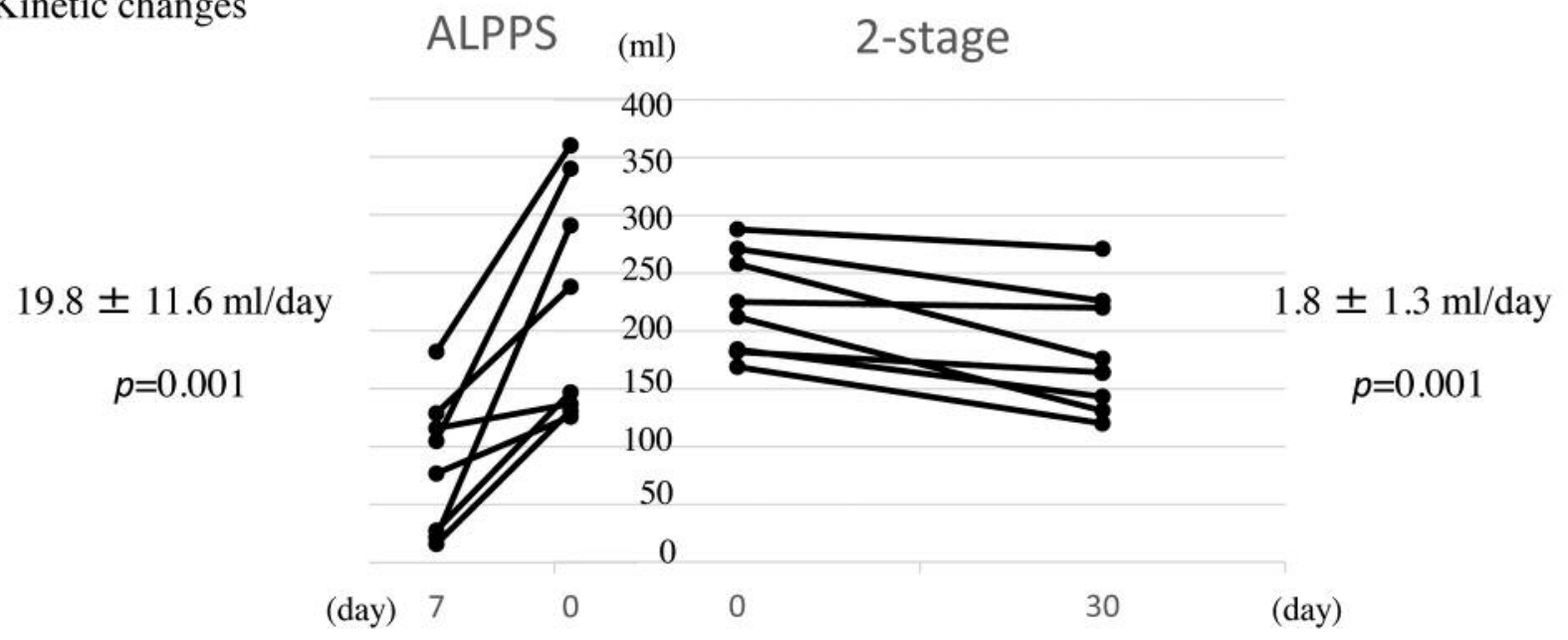

Figure 2. Change in volume, kinetic change and functional volume in the ventral right paramedian sector. Continuous lines, associating liver partition with portal vein ligation for staged hepatectomy (ALPPS) group; broken lines, classical 2-stage (CTS) group (a and c). Kinetic changes were calculated from serial liver volumes of each patient in both groups $(b)$. Pre, Preoperative; post, postoperative. $* * p<0.01$.

\section{Discussion}

A prospective study might be needed to confirm differences between ALPPS with and without MHV ligation. This represents a limitation of the present retrospective analysis. In addition to the presence or absence of congestion from the MHV ligation, another difference between ALPPS and CTS hepatectomy is that CTS hepatectomy, which does not involve liver partition, leaves open the possibility of formation of new intrahepatic collateral vessels within S4. However, we mainly compared volumes of the ventral RPS in the rt. hemiliver, while anatomic observations exclude vascular communications at the main portal fissure (8). Even assuming some formation of vascular collaterals, fewer intrahepatic collaterals have been found in PVE performed during CTS hepatectomy than in PVL during CTS hepatectomy (19). In light of such informations, our present results are important to understand the effect of congestion from MHV ligation on volume change.

Among factors likely to influence early FLR regeneration, our present findings support the hypothesis that the area of congestion in the deportalized liver provides an important 
a. volume

(ml) 450

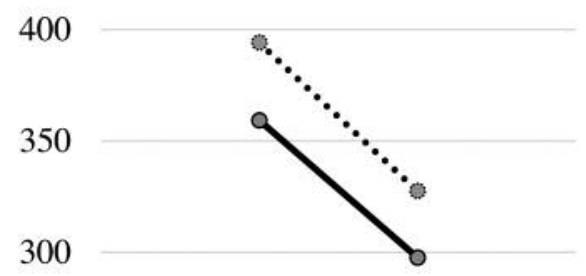

250

\begin{tabular}{lcc}
200 & pre & post \\
2-staged & $394.1 \pm 87.7$ & $327.5 \pm 114.2$ \\
\hline ALPPS & $359.2 \pm 118.1$ & $297.6 \pm 62.9$
\end{tabular}

\section{c. Functional volume}

(\%) 45

40

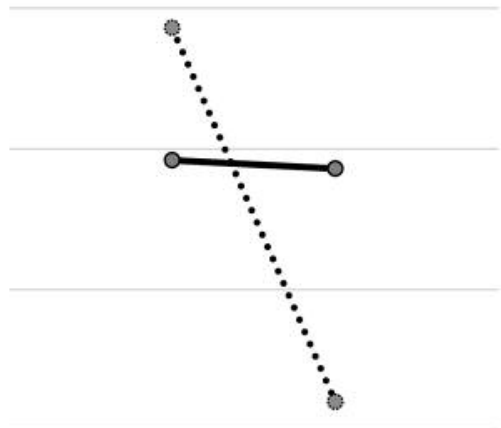

30

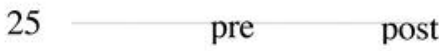

\begin{tabular}{lll} 
2-staged & $44.3 \pm 7.5$ & $31.0 \pm 12.7$ \\
\hline ALPPS & $39.6 \pm 8.8$ & $39.3 \pm 11.3$
\end{tabular}

b. Kinetic changes

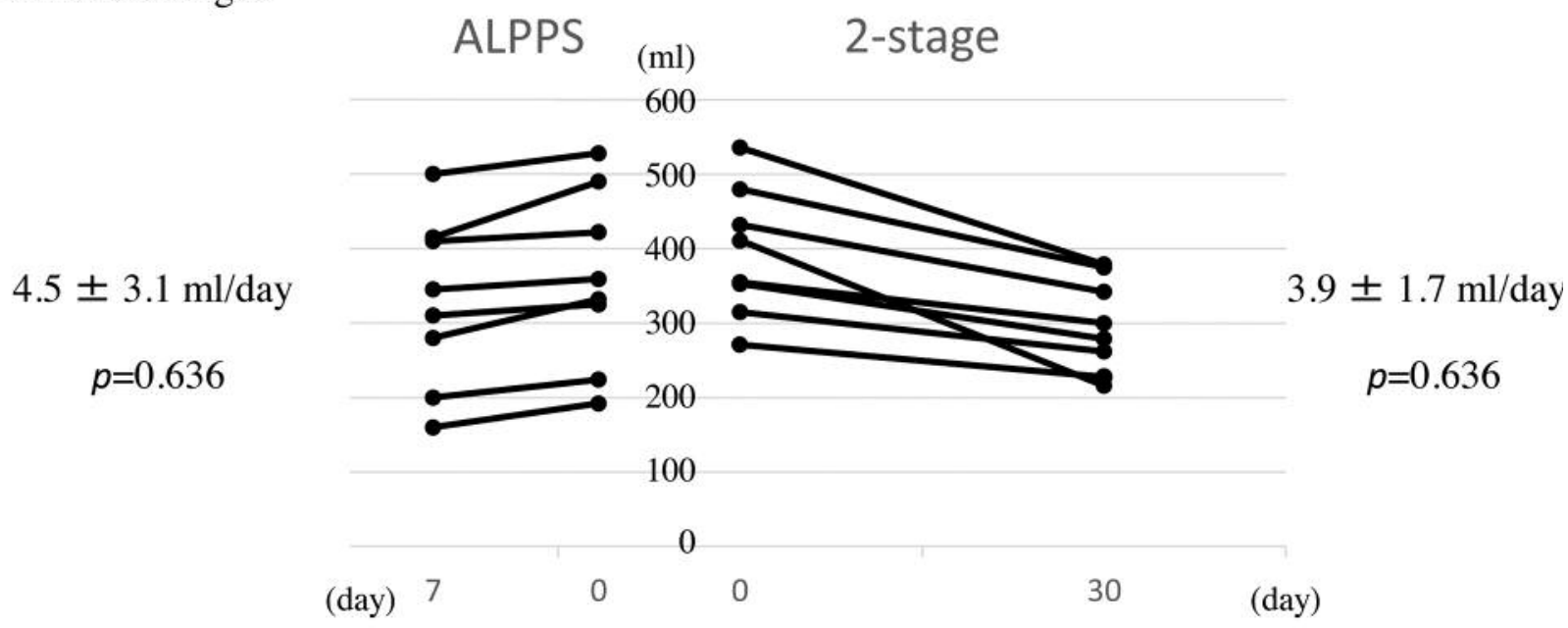

Figure 3. Change in volume, kinetic change and functional volume in the dorsal right paramedian sector plus right lateral sector. Continuous lines, associating liver partition with portal vein ligation for staged hepatectomy (ALPPS) group; broken lines, classical 2-stage (CTS) group (a and c). Kinetic changes were calculated from serial liver volumes of each patient in both groups (b). Pre, Preoperative; post, postoperative.

regenerative stimulus during ALPPS treatment. Volume and kinetic growth of the FLR have been reported to increase more dramatically in the ALPPS treatment than in CTS hepatectomy $(1,2,11,12)$. Further, when changes of volume in the nonFLR area were analyzed, the volume of the ventral RPS, which showed congestion with consequent atrophy caused by interruption of the draining veins, decreased dramatically in the ALPPS group. This volume reduction tended to be greater than in the CTS group. As opposed to the ventral RPS, no significant difference of the volume reduction in the dorsal RPS+RLS was observed between groups.
Complete devascularization of an area, such as S4 in rt. trisectorectomy, represents a strong stimulus leading to rapid and marked hypertrophy of the FLR during ALPPS (13). However, such a devascularized ischemic area can become a nidus for life-threatening sepsis (4, 13-15). Therefore, although ALPPS fosters a rapid regenerative response, it raises concern about increased morbidity and mortality (3, $11,14)$. The most frequent cause of such mortality has been infectious complications arising from the necrotic deportalized area. To perform ALPPS safely, then, surgical procedures resulting in a massive devascularized ischemic 
area, such as partitioning the liver at the falciform ligament, should be avoided. According to a previous report (15), minimizing dissection of the hepatoduodenal ligament could avoid division of direct vascular supply to S4. However, S4 always becomes avascular when $\mathrm{S} 4$ portal pedicles are divided. Also, the magnitude of FLR hypertrophy might be smaller when production of a devascularized ischemic area is avoided entirely at the first hepatectomy. One modification sometimes used is removal of the ischemic area after dividing the liver (14). The present results suggest an attractive alternative, liver partition within S4 rather than at the plane of the falciform ligament, thus dividing the liver at a point closer to the main portal fissure. This strategy aims to reduce the extent of the completely necrotic area. The resulting FLR volume is likely to be greater than that following division at the plane of falciform ligament with somewhat less hypertrophy of the FLR. Our principal intent is to avoid creation of a large completely necrotic area. MHV ligation and division at this site induces congestion and subsequent atrophy within the deportalized liver, resulting in rapid, marked hypertrophy of the FLR.

A previous study (16) using intraoperative Doppler ultrasonography showed that some $80 \%$ of patients had no anastomosis between major hepatic veins. After venous interruption, functional intrahepatic anastomoses between hepatic veins formed gradually; however, at 7 days, only about $40 \%$ of patients showed collateral formation. Producing congestion and atrophy in the deportalized liver by interruption of draining veins can, therefore, be an effective way to induce early FLR hypertrophy after the first procedure in ALPPS.

Volumetric evaluation of the FRL may be less than fully informative concerning functional reserve. In our study, the increase in FLR volume 1 week after the first procedure in ALPPS was similar to that seen for CTS hepatectomy 4 weeks after the first procedure. However, the increase of functional FLR calculated using ${ }^{99 \mathrm{~m}} \mathrm{Tc}-\mathrm{GSA}$ scintigraphy SPECT/CT in the ALPPS group tended to be smaller than that in the CTS group. We previously found the functional volume recovery after the ALPPS procedure to be smaller than the morphologic volume recovery (17). Functional change fell short of morphologic change in both the ventral RPS and the dorsal RPS+RLS area. However, the magnitude of reduction in both morphologic and functional volume after MHV division in the ventral RPS was greater than in the dorsal RPS+RLS area in ALPPS.

In conclusion, this study provides important new information concerning the significance of the congested area in the ALPPS procedure for stimulating volumetric and functional recovery. The retrospective methodology imparts a bias in selection of patients in each group. As venous return varies considerably between individuals, volume of the ventral RPS also is variable. Therefore, a prospective study might be needed to confirm the difference between ALPPS with and without MHV ligation. Nonetheless, our study provides a basis for further investigation of ALPPS. We consider the area of congestion to be clinically important for the ALPPS procedure, by stimulating regeneration, as the congested state can contribute to reduction of mortality.

\section{Financial Disclosures}

The Authors have no financial disclosures.

\section{References}

1 Schnitzbauer AA, Lang SA, Goessmann H, Nadalin S, Baumgart J, Farkas SA, Fichtner-Feigl S, Lorf T, Goralcyk A, Hörbelt R, Kroemer A, Loss M, Rümmele P, Scherer MN, Padberg W, Königsrainer A, Lang H, Obed A and Schlitt HJ: Right portal vein ligation combined with in situ splitting induces rapid left lateral liver lobe hypertrophy enabling 2-staged extended right hepatic resection in small-for-size settings. Ann Surg 255: 405414, 2012.

2 de Santibañes E and Clavien PA: Playing Play-Doh to prevent postoperative liver failure: the "ALPPS" approach. Ann Surg 255: 415-417, 2012.

3 Alvarez FA, Ardiles V, Sanchez Claria R, Pekolj J and de Santibañes E: Associating liver partition and portal vein ligation for staged hepatectomy (ALPPS): tips and tricks. J Gastrointest Surg 17: 814-821, 2013.

4 Tanaka $\mathrm{K}$ and Endo I: ALPPS: Short-term outcome and functional changes in the future liver remnant. Ann Surg 262: e88-89, 2015.

5 Tanaka K, Matsumoto C, Takakura H, Matsuo K, Nagano Y, Endo I, Togo S, Shimada H, Bourquain H and Peitgen HO: Technique of right hemihepatectomy preserving ventral right anterior section guided by area of hepatic venous drainage. Surgery 147: 450-458, 2010.

6 Cho A, Okazumi S, Makino H, Miura F, Shuto K, Mochiduki R, Tohma T, Kudo H, Matsubara K, Gunji H, Yamamoto H, Ryu M and Ochiai T: Anterior fissure of the right liver - the third door of the liver. J Hepatobiliary Pancreat Surg 11: 390-396, 2004.

7 Jaeck D, Oussoultzoglou E, Rosso E, Greget M, Weber JC and Bachellier P: A two-stage hepatectomy procedure combined with portal vein embolization to achieve curative resection for initially unresectable multiple and bilobar Colorectal Liver Metastases. Ann Surg 240: 1037-1049, 2004.

8 Couinaud C: Surgical anatomy of the liver revisited. Paris, C. Couinaud, pp. 173-177, 1989.

9 Dindo D, Demartines N and Clavien PA: Classification of surgical complications: a new proposal with evaluation in a cohort of 6336 patients and results of a survey. Ann Surg 240: 205-213, 2004.

10 Rahbari NN, Garden OJ, Padbury R, Brooke-Smith M, Crawford M, Adam R, Koch M, Makuuchi M, Dematteo RP, Christophi C, Banting S, Usatoff V, Nagino M, Maddern G, Hugh TJ, Vauthey JN, Greig P, Rees M, Yokoyama Y, Fan ST, Nimura Y, Figueras J, Capussotti L, Büchler MW and Weitz J: Posthepatectomy liver failure: A definition and grading by the International Study Group of Liver Surgery (ISGLS). Surgery 149: 713-724, 2011. 
11 Li J, Girotti P, Königsrainer I, Ladurner R, Königsrainer A and Nadalin S: ALPPS in right trisectionectomy; a safe procedure to avoid postoperative liver failure? J Gastrointerest Surg 17: 956961,2013

12 Sala S, Ardiles V, Ulla M, Alvarez F, Pekolj J, and de Santibañes E: Our initial experience with alpps technique: encouraging results. Updates Surg 64: 167-172, 2012.

13 Ratti F, Cipriani F, Gagliano A, Catena M, Paganelli M and Aldrighetti L: Defining indications to alpps procedure: technical aspects and open issues. Updates Surg 66: 41-49, 2014.

14 Andriani OC: Long-term results with associating liver partition and portal vein ligation for staged hepatectomy (ALPPS). Ann Surg 256: e5, 2012.

15 Hernandez-Alejandro R, Bertens KA, Pineda-Solis $\mathrm{K}$ and Croome KP: Can we improve the morbidity and mortality associated with the associating liver partition with portal vein ligation for staged hepatectomy (ALPPS) procedure in the management of colorectal liver metastases? Surgery 157: 194201, 2015.

16 Sano K, Makuuchi M, Miki K, Maema A, Sugawara Y, Imamura $\mathrm{H}$, Matsunami H and Takayama T: Ealuation of hepatic venous congestion: Proposed indication criteria for hepatic vein reconstruction. Ann Surg 236: 241-247, 2002.
17 Tanaka K, Matsuo K, Murakami T, Kawaguchi D, Hiroshima Y, Koda K, Endo I, Ichikawa Y, Taguri $M$ and Tanabe M: Associating liver partition and portal vein ligation for staged hepatectomy (ALPPS): Short-term outcome, functional changes in the future liver remnant, and tumor growth activity. Eur J Surg Oncol 41: 506-512, 2015.

18 Couinaud C. Recherches sur la chirurgie du confluent biliaire superieur et des canaux hepatiques. Presse Med 63: 669-674, 1955.

19 van Lienden KP, Hoekstra LT, Bennink RJ and van Gulik TM: Intrahepatic left to right portoportal venous collateral vascular formation in patients undergoing right portal vein ligation. Cardiovasc Intervent Radiol 36: 1572-1579, 2013.
Received June 27, 2016

Revised July 13, 2016

Accepted July 14, 2016 